\title{
REMUNERACIÓN VARIABLE, POLÍTICA SALARIAL Y NEGOCIACIÓN COLECTIVA: HACIA UN NUEVO ENFOQUE*
}

\author{
Rosa García Hernández \\ UAB, Universitat Autónoma de Barcelona, Departament d'Economia Aplicada \\ UB, Universitat de Barcelona, Departament d'Econometria, Estadística i Economia Aplicada
}

Orcid: 0000-0001-6670-5749

DOI: $10.1387 /$ lan-harremanak. 17494
Recibido el 30 de septiembre de 2016

Aceptado el 20 de octubre de 2016

\section{ABSTRACT}

Resumen: El objetivo de este artículo es analizar la implantación de un elemento de flexibilidad salarial, como son los sistemas de remuneración variable, y ver su conexión con las diferentes tipologias de negociación colectiva y con la politica salarial implementada por diferentes paises, a nivel de establecimiento o empresa. A partir de los datos procedentes de la European Company Survey y de diversas clasificaciones $y$ bases de datos (ICTWSS, Eurofound y Structure of Earnings Survey) y a partir de una muestra de diferentes paises europeos vemos en qué tipo de regimenes se utilizan más o menos estos esquemas de retribución variables.

Las conclusiones que se extraen es que a medida que los países tienen esquemas de negociación colectiva más descentralizada, tienen más tendencia a utilizar los sistemas de retribución variable. Pero en el caso de los esquemas de participación en beneficios y de propiedad de acciones de la empresa, los paises con esquemas de negociación

* Ponencia con la que participó en la Mesa redonda: Perspectiva profesional de los fundamentos económico-jurídicos para abordar la negociación colectiva y el control sindical económico, en el II Encuentro de Profesionales del Asesoramiento Laboral y Social, celebrado en la Facultad de Relaciones Laborales y Trabajo Social (UPV/EHU-Leioa) el día 30 de septiembre de 2016. 
colectiva más centralizada son los que tienen un peso mayor. Por tanto, se podría dar un nuevo enfoque a algunos de estos esquemas de retribución variable si las organizaciones sindicales, a cambio de aceptarlos, consiguieran más información, más control, más participación en los órganos de decisión y en las decisiones estratégicas de la empresa.

Palabras clave: sistemas de retribución variable, negociación colectiva, niveles negociación salarial, politica salarial, participación financiera trabajadores

JEL clasificación: J33, J52, J53, J54

Abstract: The aim of this article is to analyze the implementation of an element of wage flexibility such as variable remuneration systems and to see their connection with the different typologies of collective bargaining and the wage policy implemented by different countries, at the level of establishment or company. From European Company Survey data and from various classifications and databases (ICTWSS, Eurofound and Structure of Earnings Survey) and from a sample of different European countries we see in which type of regimes are used more or less these variable remuneration schemes.

The conclusions drawn are that as countries have more decentralized collective bargaining schemes, they are more likely to use variable remuneration schemes. But in the case of participation in profits and ownership of company shares, countries with more centralized collective bargaining schemes are those that have greater weight. So, a new approach could be given to some of these variable pay schemes, if trade unions, in exchange for accepting them, would gain more information, more control, more participation in the decision-making bodies and in the strategic decisions of the company.

Keywords: variable pay systems, collective bargaining, wage bargaining levels, wage policy, employee financial participation

JEL classification: J33, J52, J53, J54 


\section{SUMARIO}

Sumario: 1. Introducción. 2. Sistemas de retribución variable. 2.1. Definición. 2.2. Clasificación. 3. Esquemas de retribución variable, regímenes de relaciones laborales y niveles de negociación salarial. 3.1. Establecimientos con esquemas de retribución variable (ECS). 3.2. Regímenes de relaciones laborales. 3.3. Regímenes de negociación salarial. 3.4. Relacionando datos de diferentes fuentes. 4. Metodología y base de datos. 5. Niveles de negociación salarial y convenios colectivos en seis países europeos. 6. Esquemas de retribución variable, regímenes de relaciones laborales y niveles de negociación salarial en el caso de seis países europeos. 7. Conclusiones a partir de los resultados. 8. Repercusiones y políticas. 9. Bibliografía.

\section{Introducción}

Si la entrada en la UEM, para los gobiernos de los países de la Eurozona, supuso la pérdida de soberanía en algunas políticas económicas, como la Política Monetaria y la Política de Tipo de Cambio, y supuso restricciones importantes en materia de Política Fiscal (reforzadas con la aprobación del Pacto de Estabilidad y Crecimiento), la crisis económica iniciada en 2008 agudizó esta situación. Implicó una ampliación de la pérdida de soberanía en aspectos relacionados con la Política fiscal, por un lado, como la consolidación fiscal y las restricciones en los niveles de gasto público y, por otro lado, con los Sistemas de bienestar, entre otros.

Toda esta coyuntura es especialmente relevante en el caso de los países de la periferia de la Eurozona, donde las condiciones impuestas para acceder a los programas de ayuda financiera de Europa han supuesto cambios profundos en las decisiones de muchas políticas económicas (González et al. 2015). Las políticas relacionadas con el mercado de trabajo no han sido una excepción y también se han visto afectadas.

En el caso de España, la Reforma Laboral de 2012 ha incidido de lleno en el sistema de relaciones laborales instaurado en la democracia, porque la negociación colectiva ha dejado de tener capacidad para incidir en el mercado de trabajo, suponiendo un debilitamiento de la capacidad de los sindicatos para influir en el contenido de los convenios (Cruz, 2015). Por un lado, rompe con el 
mantenimiento del equilibrio entre las partes de la relación laboral y, por otro lado, con la tradición pactista y de diálogo, dando mucho más poder a las empresas. Las consecuencias han sido la reducción salarial, un empeoramiento de las condiciones de trabajo y un aumento de la precariedad laboral. (Ruesga, 2015). Uno de los aspectos introducidos por esta Reforma Laboral ha sido la prioridad para que las empresas se acojan a los convenios de empresa frente a los convenios de nivel superior. El otro aspecto es que se introduce la posibilidad de que las empresas con dificultades se puedan «descolgar» de los convenios, es decir, que puedan dejar de cumplir algunos de los aspectos pactados.

El hecho de que se haya introducido la posibilidad de descentralizar más la negociación colectiva tiene repercusiones, entre otros aspectos, en el ámbito de la política salarial.

$\mathrm{Y}$ es en este nivel en el que hemos de situar a los sistemas de remuneración variable como un elemento de flexibilización salarial y que han visto crecer su importancia en los últimos años por diferentes motivos: por un lado, debido a su importancia creciente en los Convenios Colectivos como una forma de pago no consolidado; por otro lado, porque ellos se implementan a toda la plantilla, no sólo a los altos ejecutivos (García, 2016).

Hemos dividido este artículo en diferentes apartados. En el apartado 2, abordamos el tema de la definición y clasificación de los sistemas de retribución variable. En el apartado 3, relacionamos los esquemas de retribución variable, con los regímenes de negociación colectiva y los niveles de negociación salarial en el caso de los 28 países de la UE, con los datos de 2013. En el apartado 4, hacemos la presentación de la base de datos SES y relacionamos los niveles de negociación salarial con los tipos de convenios colectivos. En el apartado 5, relacionamos los niveles de negociación salarial con los tipos de convenios colectivos en el caso de seis países europeos, con los datos de las tres olas de la SES: 2002, 2006 y 2010. En el apartado 6, relacionamos los esquemas de retribución variable, los regímenes de negociación colectiva y los niveles de negociación salarial en el caso de estos seis países europeos, con los datos de 2016. En el apartado 7 , resumimos las principales conclusiones y en el apartado 8 , analizamos las repercusiones y las políticas posibles a aplicar.

\section{Sistemas de retribución variable}

\subsection{Definición}

En los sistemas de retribución fija los empleados reciben una cantidad de dinero fija mensualmente o anualmente, según viene estipulado en el convenio colectivo firmado (en función de la categoría, número de horas trabajadas, etc.). Por oposición, los sistemas de retribución variable implican que el sueldo reci- 
bido no siempre es el mismo y que hay complementos salariales que se añaden a la parte de salario regular o fijo, conectados, por ejemplo, con la evolución de los objetivos de la empresa o del grupo empresarial (en términos de beneficio, ventas, productividad, etc.) o con la evolución de objetivos a nivel individual (productividad, absentismo, etc.). Estos esquemas normalmente no substituyen al salario base, pero sí se pueden utilizar para compensar reducciones de éste, en algunas ocasiones.

Tradicionalmente, los esquemas de retribución variable han existido en diferentes formas, como los salarios a destajo o las bonificaciones o comisiones de los comerciales en algunos sectores. También han existido los complementos salariales vinculados por ejemplo con la productividad. Y también hay que mencionar las retribuciones variables de los altos ejecutivos vinculadas a los resultados de la empresa, por ejemplo.

Estos esquemas de remuneración variable han tenido una importancia creciente durante los últimos ańos, como diferente literatura muestra (Pendleton et al., 2009). Podemos hablar de un resurgimiento de estos esquemas a partir de los años 80 y 90, que es cuando la popularidad de estos esquemas es mayor, porque son vistos como una posible solución a la recesión económica en aquellos ańos, particularmente en países como USA y UK (Gilman, 1998).

La globalización de la producción y el aumento de la competencia en los mercados de productos son algunos elementos que explicarían el cambio en la utilización de la retribución variable en algunos países (Pendleton et al., 2009). Se trataría de transferir una parte del riesgo a los trabajadores y de proporcionarles incentivos con el objetivo de mejorar su rendimiento (Brown y Heywood, 2002; Drago y Heywood, 1995). Otro punto importante sería la disminución de los costes relacionada con el debilitamiento de la fuerza de los sindicatos y con el diseño de formas sofisticadas para medir el esfuerzo y el resultado de los empleados (Pendleton et al., 2009).

Cabe destacar que, en la crisis económica iniciada en 2008, uno de los problemas también fue la remuneración variable que se daba a algunos trabajadores del sector bancario de USA, premiando la colocación de las llamadas «hipotecas subprime» entre sus clientes.

Hay diferentes elementos que nos permitirían hablar del resurgimiento de estos esquemas con rasgos nuevos. En primer lugar, tendríamos la introducción y la importancia de formas de retribución variable relacionados con la Participación financiera de los empleados en la empresa, a modo de participación en los beneficios de la empresa y a modo de la Propiedad de acciones de la empresa. En segundo lugar, habría el hecho que estaríamos frente a esquemas que no sólo afectan a determinados cargos directivos sino que están afectando a toda la plan- 
tilla de la empresa. Y, en tercer lugar, otro elemento a destacar sería el carácter no consolidado de estos pagos y complementos ${ }^{1}$ (García, 2016).

\subsection{Clasificación}

Variable Pay in Europe fue el primer estudio sobre Sistemas de Retribución Variable publicado por EIRO (European Industrial Relations Observatory) a nivel europeo. En este estudio se hace una clasificación de los esquemas de retribución variable en tres categorías diferentes (Van Het Kaar and Grünell, 2001):

- Pago por resultados

- Pago por rendimiento

— Esquemas de participación financiera de los trabajadores (participación en beneficios y propiedad de acciones).

Otros estudios y artículos posteriores (Pendleton et al., 2009) (Pouliakas et al., 2011) han profundizado en esta clasificación, teniendo en cuenta dos elementos:

- Si el esquema de remuneración tiene relación con objetivos colectivos de la empresa o si tiene relación con objetivos individuales del trabajador

— Si el esquema de remuneración supone una evaluación objetiva o una evaluación subjetiva

A partir de estos elementos se plantean otras clasificaciones como la que se utiliza en el European Company Survey más desglosadas: pago por resultados, pago por rendimiento individual., pago por rendimiento colectivo o en equipo, participación en beneficios y propiedad de acciones.

\section{Esquemas de retribución variable, regímenes de relaciones laborales y niveles de negociación salarial}

\subsection{Establecimientos con esquemas de retribución variable (ECS)}

La European Company Survey (ECS) es una encuesta elaborada por Eurofound (European Foundation for the improvement of living and working conditions) en la cual, a partir de una muestra representativa de establecimientos, se

\footnotetext{
1 Se refieren a aquellos pagos únicos hechos a los empleados. Estos pagos no se actualizaran según las tablas referenciadas en los convenios, como el resto del salario. Y no contribuirán en el cálculo para las pensiones por jubilación.
} 
entrevista al gerente responsable de los recursos humanos y a representantes de los trabajadores. A partir de los datos de la European Company Survey (ECS 2013) $)^{2}$, podemos observar cómo un $63 \%$ de los establecimientos europeos analizados utilizaban algún tipo de sistema de retribución variable. Un $43 \%$ de los establecimientos utilizaban esquemas de pagos por rendimientos individuales, un $34 \%$ de los establecimientos utilizaban esquemas de pago por resultados y un $30 \%$ utilizaban esquemas de participación en los beneficios.

\subsection{Regímenes de relaciones laborales}

Podemos agrupar a los 28 países miembros de la UE en 5 clusters de regímenes de relaciones laborales (ETUI, 2012) (European Commission, 2009):

1. Norte de Europa: Dinamarca, Finlandia y Suecia

2. Europa Central-Occidental: Austria, Bélgica, Alemania, Luxemburgo, Holanda y Eslovenia

3. Europa del Sur: Francia, Grecia, Italia, Portugal y España

4. Europa Liberal-Occidental o Anglo-Sajona: Chipre, Irlanda, Malta y el RU

5. Europa Central-Oriental: Bulgaria, República Checa, Estonia, Hungría, Letonia, Lituania, Polonia, Rumania, Eslovaquia y Croacia ${ }^{3}$.

Estos cinco grupos se diferencian unos de otros en algunos elementos como el grado de densidad salarial., el nivel predominante de negociación colectiva, la existencia de salario mínimo obligatorio o el papel del Estado en la negociación colectiva, entre otros. (ETUI, 2012).

En el caso del clúster del Norte de Europa, el de la Europa del Sur y el de la Europa Central-Occidental., se observa un tipo de negociación colectiva a varios niveles (multi-employer) con varios empleadores, entre los sindicatos y asociaciones de empresarios El tipo de negociación colectiva más frecuente es la de nivel sectorial.

En el caso de los países que forman parte del clúster de la Europa Anglosajona y de la Europa Central-Oriental., la negociación colectiva es con un solo empleador (single-employer), entre empresarios y sindicatos. El tipo de negociación colectiva más frecuente es la de nivel de empresa.

En los países del Norte y Europa Central-Oeste se establece una negociación a tres bandas, entre los actores políticos y los sindicatos y asociaciones de empresarios. En los países que pertenecen al modelo anglosajón, el funcionamiento es

\footnotetext{
${ }^{2}$ La ECS 2013 incluyó 32 países: los 28 miembros de la Unión Europea, la Antigua República Yugoslava de Macedonia, Islandia, Montenegro y Turquía.

3 Se adhiere a la UE en el año 2013.
} 
distinto, y la influencia de los interlocutores sociales no siempre está clara, porque no se refleja en los resultados de las políticas. En el sur de Europa, la participación de los interlocutores sociales en la política depende de la voluntad de los gobiernos. En Europa Central y del Este, la politización de los interlocutores sociales limita su influencia en la formulación de políticas.

En el clúster del Norte de Europa, el de la Europa Anglo-sajona y el de la Europa Occidental., la participación del Estado no es frecuente. En la Europa del Sur, el Estado influencia los resultados de la negociación colectiva de manera indirecta (ETUI, 2012).

\subsection{Regímenes de negociación salarial}

Las variables que se utilizan para clasificar los regímenes de negociación salarial habitualmente son dos. Por un lado, tenemos el grado de coordinación de la negociación salarial. Esta variable se basa en un conjunto de expectativas sobre las características institucionales en la determinación salarial que pueden incidir en el hecho que haya más o menos coordinación (Kenworthy, 2001). Por otro lado, tenemos el nivel de negociación salarial., que hace referencia al nivel predominante ${ }^{4}$ en el cual la negociación salarial tiene lugar.

La base de datos Institutional Characteristics of Trade Unions, Wage Setting, State Intervention and Social Pacts (ICTWSS 4.0) es una serie temporal diseñada por J.Visser y alojada por parte del Amsterdam Institute for Advanced Labour Studies (AIAS) que muestra una gran colección de variables y de indicadores de relaciones laborales para países de la UE y de la OCDE (Eurofound, 2014).

Según la base de datos ICTWSS (Visser, 2013), podemos hacer la siguiente clasificación de los niveles de negociación salarial:

5. Negociación colectiva a nivel central o a nivel sectorial

4. Negociación colectiva entre el nivel central y el nivel sectorial

3. Negociación colectiva a nivel sectorial

2. Negociación colectiva entre el nivel sectorial y el nivel de empresa

1. Negociación colectiva a nivel local o a nivel de empresa

\subsection{Relacionando datos de diferentes fuentes}

Si cruzamos los datos de la European Company Survey (ECS, 2013) con los datos de los clúster de los regímenes de negociación colectiva (ETUI, 2012)

\footnotetext{
4 Un nivel es predominante si cubre al menos dos terceras partes de la tasa de cobertura de la negociación total en un año y país determinado. Si cubre menos pero más de una tercera parte, se considera una situación mixta o una situación intermedia entre dos niveles (Visser, 2013).
} 
(European Commission, 2009) y los datos de los regímenes de negociación salarial (ICTWSS 5.0, 2015), podemos ver la importancia de los diferentes esquemas de remuneración variable en función del país, del clúster al cual pertenecen y del tipo de negociación salarial.

En primer lugar podemos decir que, en el caso del pago por resultados, a excepción de Austria (53 \%) que tiene un nivel de negociación salarial intermedio (nivel 3), la mayoría de países que muestran porcentajes más elevados (superiores o cercanos al $50 \%$ ) tienen niveles de negociación salarial descentralizados (nivel 1). Este sería el caso de países como Lituania,(72,2\%), República Checa $(57,9 \%)$ y Estonia $(56,9 \%)$ pertenecientes al clúster de la Europa CentralOriental.

En segundo lugar, tendríamos un grupo de países con un nivel de negociación salarial intermedio (nivel 3) con porcentajes elevados de establecimientos con pagos por rendimiento individual. Éste sería el caso de Dinamarca $(53,4$ \%) del clúster del Norte de Europa, y Austria (56,4 \%) y Eslovenia (72,5 \%) del clúster de la Europa Central-Occidental. El resto de países con porcentajes más elevados formarían parte del clúster Europa Central-Oriental con un nivel de negociación salarial descentralizado (nivel 1 y nivel 2 para el caso de Eslovaquia): Polonia (54,6\%), Eslovaquia (55,2 \%), República Checa (74,3\%), Estonia $(53,6 \%)$ y Lituania $(67,2 \%)$.

En tercer lugar, en el caso de los esquemas de pago por rendimiento de equipo los porcentajes son en general más bajos en todos los casos. Los países con niveles más elevados que se acercarían al 50 \% serían, por un lado, Eslovenia $(47,9 \%)$ con un nivel de negociación salarial intermedio (nivel 3). Y, por otro lado, tendríamos paises pertenecientes al clúster de Europa Central-Oriental., como Estonia (49,1 \%) y Lituania $(47,6 \%)$, los dos con un nivel de negociación salarial descentralizado (nivel 1).

En cuarto lugar, en relación a los países con porcentajes más elevados de establecimientos con esquemas de participación en beneficios, tenemos que destacar el caso de Finlandia $(50,8 \%)$ con un nivel de negociación salarial centralizado (nivel 5). Al mismo tiempo encontramos el caso de Eslovenia (55,4 \%) con un nivel de negociación salarial intermedio (nivel 3), Eslovaquia (53,1 \%) (nivel 2) y la República Checa $(50,5 \%)$ y Lituania $(53,1 \%)$ con un nivel de negociación salarial descentralizado (nivel 1).

Y finalmente, en el caso de la propiedad de acciones los porcentajes de establecimientos de todos los paises son muy bajos: en ningún caso se llega al $13 \%$. Aquí cabe destacar a países como Finlandia $(12,5 \%)$ con un nivel 5 de negociación salarial y Suecia $(9,2 \%)$ y Francia $(8,1 \%)$ con un nivel 3 de negociación salarial. Y también países con un nivel de negociación salarial 1 como es el caso del Reino Unido (8,6 \%) y Lituania (12,8 \%). 


\section{Metodología y base de datos}

La base de datos que hemos utilizado es la Stucture of Earnings Survey (SESmicrodata) (Encuesta de Estructura Salarial) de Eurostat para tres diferentes olas (2002, 2006 y 2010) (Eurostat-Research Proposal 53/2015-SES) ${ }^{5}$. La SES es una encuesta que relaciona las características de una muestra de empresas o establecimientos ${ }^{6}$ con las características de una muestra de asalariados que trabajan en ellas ${ }^{7}$. Incluye información sobre los asalariados que han contribuido al sistema de seguridad social en todo el mes de octubre del ańo de referencia. Es una encuesta que se realiza cada cuatro años desde 1995, que ofrece un conjunto de datos de corte transversal (cross-section) y que contiene microdatos empleadorempleado (observaciones de varios trabajadores empleados en cada establecimiento) (Ramos, Sanromà, Simon, 2014).

En nuestra base de datos la variable «Convenio Colectivo» identifica el tipo de convenio colectivo que cubre al menos el $50 \%$ de los empleados de cada planta productiva o fábrica. Algunas de las diferentes opciones de esta variable son:

- Convenio colectivo a nivel nacional

- Convenio colectivo a nivel sectorial

- Convenio colectivo para sectores individuales en regiones individuales

- Convenio colectivo a nivel de empresa o a nivel de empresario individual

- Convenio colectivo a nivel de planta productiva o fábrica

Para hacer nuestro análisis combinamos la clasificación de convenios colectivos de la encuesta de SES, con la clasificación de niveles en la negociación salarial de la base de datos ICTWSS 4.0 (Visser, 2013) y la que utiliza Eurofound (Eurofound, 2014).

En función de si tenían información desglosada sobre los esquemas de retribución variable y con el objetivo de tener al menos un país para cada tipo de nivel de negociación salarial., seleccionamos a seis países europeos: Finlandia, España, Portugal, Francia, Rumanía y Polonia. No pudimos obtener datos correspondientes a todos los 5 clústers de regímenes de relaciones laborales.

5 Proyecto de investigación del Departamento de Econometría, Estadística y Economía Aplicada de la UB «Análisis y Evaluación de políticas públicas: Metodologías y aplicaciones empíricas» ECO 2016 76866_R del Ministerio de Economía y Competitividad.

${ }^{6}$ Empresas o establecimientos de más de 10 trabajadores pertenecientes a todos los sectores de actividad económica según (NACE), con excepción de la administración pública.

7 Se hace un muestreo en dos etapas. En la primera etapa se diseña una muestra aleatoria estratificada de unidades locales (establecimientos) o empresas. En la segunda etapa se diseńa una muestra aleatoria estratificada de empleados tomados de las unidades locales (establecimientos) o empresas escogidos. 


\section{Niveles de negociación salarial y convenios colectivos en seis países europeos}

En este apartado recogemos información correspondiente a los seis países seleccionados procedente de las tres olas de la SES analizadas y la unimos a la información previa de los niveles de negociación salarial que hemos obtenido de ICTWSS y de Eurofound.

Con toda esta información, en primer lugar, podemos observar la información detallada por países del peso que tienen los diferentes tipos de convenios colectivos en el total de observaciones y en cada una de las tres olas de la SES. En el caso de Finlandia, con un nivel de negociación salarial que pasa del 5 al 3 entre la SES 2002 y la SES 2010, la mayoría de las observaciones (98 \%) muestran convenios colectivos negociados a nivel nacional en las tres olas. En España, que se mantiene a lo largo de las tres olas en un nivel de negociación salarial de 4, la mayoría de las observaciones muestran la importancia de los convenios colectivos negociados a nivel sectorial y a nivel regional y el incremento del peso de los convenios de empresa. En Portugal., que es un país que se mantiene en el nivel 3 de negociación salarial., podemos observar el peso de los convenios colectivos a nivel nacional., a nivel sectorial y a nivel de empresa. En el caso de Francia, con un nivel de negociación salarial de 2 a lo largo de las tres olas, en el 2002 se observa una gran importancia de los convenios colectivos a nivel nacional., en el 2006 los convenios sectoriales son los más frecuentes y en el 2010 los convenios sectoriales a nivel regional son los que destacan, juntamente con un aumento de peso de los convenios de empresa. En Rumanía que, en 2002 y 2006 mostraba un nivel de negociación salarial de 2 y en 2010 pasa a tener un nivel de negociación salarial de 3, se observa un protagonismo de los convenios de empresa y los convenios negociados a nivel nacional. Finalmente, En Polonia, con un nivel de negociación salarial de 1 , se observa una clara importancia del papel de los convenios de empresa. Por tanto, podemos decir que hay una cierta relación entre el nivel de negociación salarial del país y el peso de determinados tipos de convenios colectivos: cuanto más centralizado es el nivel de negociación salarial más importancia de los convenios a nivel nacional o sectorial y cuanto más descentralizado es el nivel de negociación salarial más importancia de los convenios de empresa.

En segundo lugar, nuestra base de datos SES ofrece información sobre la variable «Bonus anuales» que nosotros utilizados como "proxy» de los esquemas de remuneración variables. Sólo para la ola de 2002 se puede encontrar el desglose de los «Bonus anuales» en: bonus regulares ${ }^{8}$, bonus por productividad y participación en beneficios.

8 Bonus por vacaciones, $13 .^{\mathrm{a}}$ y $14 .^{\mathrm{a}}$ pagas y las comisiones ocasionales. 
En el caso de los países analizados podemos concluir que aquellos que en 2002 tenían un nivel de negociación salarial más centralizado (niveles 5, 4 e incluso 3) muestran un porcentaje más elevado de observaciones con bonos regulares respecto al resto de tipos de bonus. En el caso de Finlandia, España y Portugal., los bonus regulares suponen el $96 \%$, $99 \%$ y $100 \%$ de las observaciones respectivamente. Hay que remarcar que el peso de las observaciones con bonus por productividad o participación en beneficios que no tengan además bonus regulares es muy bajo. Es decir, que siempre que se cobra un bonus por productividad o participación en beneficios además se está cobrando el bonus regular. En el caso de Francia, Rumanía y Polonia, con una negociación salarial más descentralizada (niveles de 2 y 1) la situación es completamente diferente. El porcentaje de observaciones con bonus regulares es más bajo y el porcentaje de observaciones con bonus por productividad o participación en beneficios y sin bonus regulares es superior, situandose entre un $20 \%$ y un $30 \%$. En el caso de Polonia, nos encontramos un caso extremo porque ninguna de las observaciones con participación en beneficios tiene además bonus regulares. Es decir que, en estos países puede ser que el trabajador o trabajadora cobre algún bonus por productividad o tenga participación en beneficios, pero esto no le asegura que cobre también el bonus regular, como en el caso de los países con niveles de negociación salarial más centralizados.

\section{Esquemas de retribución variable, regímenes de relaciones laborales y niveles de negociación salarial en el caso de seis países europeos}

Ahora repetiremos el ejercicio de cruzar los datos de la European Company Survey (ECS, 2013) con los datos de los clústers de los regímenes de negociación colectiva (ETUI, 2012) (European Commission, 2009) y los datos de los regímenes de negociación salarial ICTWSS versión 5.0 (Visser, 2015) , pero en el caso de nuestros seis países europeos.

A partir de aquí podemos afirmar que los países con unos regímenes de negociación salarial más descentralizada (Polonia y Rumanía) o con un tipo de negociación salarial más centralizada (Finlandia) son aquellos países que muestran

9 Los mayores cambios entre la versión 5.0 de la base de datos ICTWSS y la versión anterior 4.0, publicada en abril de 2013, se encuentran en las secciones sobre la fijación de salarios y el «nivel predominante de la negociación", que se define como el nivel al que se aplican la mayoría (dos tercios o más, en términos de cobertura) de convenios colectivos. En la versión 5.0 un nuevo conjunto de variables intenta capturar el nivel real de la negociación sobre salarios y horas de trabajo a través de un nuevo conjunto de variables: la frecuencia o el alcance de la negociación; la articulación de la negociación de varios niveles: la base legal o contractual de excepción: las cláusulas generales de apertura en los acuerdos sectoriales, y cláusulas de rigor relacionados con la crisis en los acuerdos de cualquier tipo. 
un porcentaje más elevado de establecimientos con esquemas de retribución variable basados en el pago por resultados, el pago por rendimiento colectivo y el pago por rendimiento individual. De esta manera, alrededor de un $42 \%$ (en términos medios) de los establecimientos de Finlandia, Rumanía y Polonia muestran esquemas de pago por resultados. Alrededor de un $48 \%$ (en términos medios) de los establecimientos de estos tres países muestran esquemas de pago por rendimientos a nivel individual. Y un $34 \%$ (en términos medios) de los establecimientos de estos tres países muestran esquemas de pago por rendimiento colectivo o en equipo.

Mientras que los países con regímenes de negociación salarial intermedia (España, Portugal y Francia) son aquellos que muestran porcentajes más bajos de establecimientos con esquemas de pago por resultados, pago por rendimiento individual y pago por rendimiento colectivo. En relación a la participación en los beneficios y a la propiedad de acciones (esquemas de participación financiera de los trabajadores en la empresa) los países que cuentan con porcentajes más elevados son Finlandia y Francia con esquemas de negociación colectiva centralizada e intermedia, respectivamente.

\section{Conclusiones a partir de los resultados}

A modo de resumen, podemos decir que, con los datos del 2013, los países con porcentajes más elevados de establecimientos que tienen esquemas de pago por resultados, pago por rendimiento y participación en beneficios, en la mayoría de casos pertenecen al clúster de Europa Central-Oriental y tienen un nivel de negociación salarial descentralizado (nivel 1). Algunos países que forman parte del clúster de Europa Central-Occidental y que tienen un nivel de negociación salarial intermedio (nivel 3) también muestran porcentajes elevados en estos tipos de modelos de retribución.

En el caso de los esquemas de participación financiera de los trabajadores, como el caso de la participación en beneficios, cabe remarcar los porcentajes altos que muestra algún país del clúster Norte de Europa con un nivel de negociación salarial centralizado (Finlandia-nivel 5) y de algún país del clúster de la Europa Central-Occidental con un nivel de negociación salarial intermedio (Eslovenia-nivel 3). En el caso de la propiedad de acciones, los países con porcentajes de establecimientos más elevados tienen un nivel de negociación salarial 5 (Finlandia) y un nivel de negociación salarial 1 (Lituania).

Estas conclusiones también las podríamos extraer al concretar nuestro análisis en la muestra de seis países europeos, con los datos de 2013. Así, los países que tienen un porcentaje más elevado de establecimientos con esquemas de retribución variable como pago por resultados o pago por rendi- 
miento, pertenecen al clúster de la Europa Central-Oriental., como es el caso de Rumanía y Polonia. También es importante destacar el papel de los países del Norte de Europa como es el caso de Finlandia, que con un nivel de negociación salarial mucho más centralizado presenta también elevados porcentajes de establecimientos. Pero donde el papel de Finlandia es más destacado, juntamente con Francia es en el de los esquemas financieros de participación de los trabajadores: participación en beneficios y propiedad de acciones. En estos casos, el papel de los países de la Europa Central-Oriental, con niveles de negociación salarial más descentralizados, es menos importante. Teniendo más importancia el papel de países con niveles de negociación salarial más centralizada.

Con los datos provenientes de las tres olas de la SES (2002,2006 y 2010), en el caso de los mismos seis países europeos, podemos afirmar (en líneas generales) que los países con niveles de negociación salarial más centralizados son aquellos que muestran un porcentaje más elevado de las observaciones con convenios colectivos a nivel nacional o sectorial. Y los países con niveles de negociación salarial más descentralizados muestran un porcentaje más elevado de las observaciones con convenios a nivel de empresa. Además, con el desglose del tipo de bonus en la ola SES-2002, se pone de manifiesto que los países con niveles de negociación salarial más centralizados muestran un porcentaje más elevado de las observaciones con bonus regulares y en la mayoría de observaciones con bonus por productividad o con participación en beneficios también se perciben los bonus regulares. En el caso de los países con niveles de negociación salarial más descentralizados, el porcentaje de observaciones con bonus regulares es menor y podemos encontrar observaciones con bonus por productividad o con participación en beneficios que no estén percibiendo los bonus regulares.

A partir de la muestra de países que hemos seleccionado, y de los datos del resto de países, podemos concluir que los países del clúster de la Europa Central-Oriental., con niveles de negociación salarial descentralizados son los que tienen un peso más importante, en líneas generales, en la utilización de les esquemas de retribución variables, como los pagos por resultados y pagos por rendimiento. En el caso de la participación en beneficios, a parte de ellos, aparecen también otros países con pesos importantes con niveles de negociación salarial más centralizados. En el caso de la propiedad de acciones, es interesante remarcar que sólo algún país del clúster de la Europa Central-Oriental tiene un papel destacable, al lado de otros países del clúster del Norte de Europa.

Por tanto, a más nivel de descentralización en la negociación colectiva y en el nivel de negociación salarial más utilización de esquemas de retribución variable, como pagos por resultados y pagos por rendimiento. Pero este comportamiento no queda tan claro en el caso de los esquemas de participación financiera 
de los trabajadores. En el caso de la participación de los beneficios y, sobretodo, en el caso de la propiedad de acciones, países con niveles de negociación colectiva y de negociación salarial más centralizados tienen un papel importante.

\section{Repercusiones y políticas}

La introducción de los esquemas de retribución variable, según diferentes teorías, tendría relación con la utilización de recompensas para motivar a los trabajadores y para mejorar su rendimiento. (Shuler, 1998). Así, los trabajadores trabajarían más duro si creen que los premios y las recompensas monetarias serán el resultado de sus esfuerzos. (Boachie-Mensah, 2011). Este punto de vista que proviene principalmente del enfoque tradicional de la Empresa y la Gestión se puede conectar con el enfoque del modelo competitivo neoclásico del mercado de trabajo, según el cual los salarios son determinados por el nivel de productividad de los trabajadores. De esta manera, los sistemas de remuneración variable serían una herramienta para mejorar el nivel de motivación de la plantilla. Y a más motivación, más productividad y más competitividad para la empresa. Sin embargo estas teorías de la motivación por retribución ofrecen una explicación muy simplista, porque ignoran elementos importantes (Kessler and Purcell, 1992). Así, las características de la organización, las condiciones ambientales, las características del trabajo y los sistemas de remuneración hacen que todo sea mucho más complejo (Perry et al. 2009). Algunos estudios son escépticos sobre el papel de los esquemas de retribución variable en introducir más motivación. de la motivación (Gilman, 1998).

Algunos de estos sistemas de pagos variables vinculan una parte de la retribución retribución al rendimiento de la empresa o al rendimiento individual de los trabajadores y trabajadoras y, ésta situación, más que incrementar el nivel de motivación y de productividad, puede generar situaciones de desigualdad salarial. (García-Hernández, 2016). Si la tendencia es ir hacia estos esquemas de flexibilidad salarial., esto implica poner a la retribución "en riesgo» (Arrowsmith et al., 2007). Toda la plantilla asume una parte del riesgo de la empresa, al mismo nivel que el empresario (Suff and Reilly, 2004), independientemente del grado de responsabilidad.

Una posible manera de compensar esta situación sería que las organizaciones sindicales consiguieran más control, más información y más democracia dentro de las empresas. De manera que si hay que asumir más riesgos, si los salarios de todos los trabajadores y trabajadoras dependen de la marcha de la empresa, a cambio se obtenga más participación en las decisiones estratégicas de la empresa.

Porque no todos los esquemas de retribución variable funcionan de la misma manera. Recibir una remuneración en función de los resultados de la 
empresa o en función del rendimiento no es lo mismo que tener una participación en los beneficios o disponer de acciones de la empresa. En estos dos últimos casos se puede pedir una contraprestación a cambio de más participación en la decisiones importantes de la empresa, por ejemplo. Y, como hemos visto, con los datos que hemos analizado, los países con esquemas de negociación colectiva más descentralizados no utilizan de la misma manera los esquemas de retribución variable. Básicamente se centran más en el pago por resultados y el pago por rendimiento, pero tienen un papel más pequeño en la propiedad de acciones.

$\mathrm{Y}$ es que si se hace un nuevo diseño de los esquemas de participación financiera, como la participación en beneficios o la propiedad de acciones, éstos podrían ser elementos que ayudaran a democratizar la organización interna de las empresas. Podrían introducir elementos de cogestión y podrían hacer que los trabajadores y trabajadoras participaran de las decisiones empresariales clave. Estos elementos podrían ser mucho más motivadores que el hecho de conectar la remuneración a los resultados de la empresa o al rendimiento, individual y/o colectivo.

Por tanto, se trataría de darle un nuevo enfoque a algunos de estos esquemas de retribución variables y que sean la excusa para hacer la organización de las empresas mucho más participativa y para frenar las políticas de contención salarial.

\section{Bibliografía}

Arrowsmith J., Nicholaisen, H. Bechter B. and Nonell. R. (2007). «The management of variable pay in banking: forms and rationale in four European countries». 8th European Congress of the International Industrial Relations Association, 2007, Manchester, UK.

Boachie-Mensah, F. (2011). «Performance-Based Pay as a Motivational Tool for Achieving Organisational Performance: An Exploratory Case Study». International Journal of Business and Management. Vol 6. N.12.

Brown, M. and HeYwood, J.S. ed. (2002). Paying for performance: an international comparison. M.E. Sharpe Inc. USA

CruZ Villalón, J. (2015): «La nueva regulación de la negociación colectiva: ruptura del equilibrio en las relaciones laborales en aras de una flexibilidad sin consenso». Temas para el debate. ISSN 1134-6574. N.o 249-250 (agosto-septiembre) 2015. Págs. 28-30.

Drago, R. and Heywood, J.S. (1995). The Choice of Payment Schemes:Australian Establishment Data. Australian Department of Industrial Relations

ETUI (2012). Benchmarking working Europe 2012. «Collective bargaining and diversity in wage developments». Brussels

Eurofound (2014). Pay in Europe in the 21st century. Publications office of the European Union. Luxembourg.

European Commission (2009) Industrial relations in Europe 2008, Luxembourg: Publication Office of European Union. 
García-Hernández, R. (2016): «Approaches to Variable Pay Systems». PhD Dissertation. Universitat de Barcelona.

Gilman, M.W. (1998). «Performance related pay in practice: organization and effect». $\mathrm{PhD}$ thesis. University of Warwick.

González Begega, S., Luque Balbona D., Guillén A.M. (2015): «Gobiernos y sindicatos ante la reforma del estado del bienestar. ¿Ruptura del diálogo social en la periferia de la eurozona?». Revista de Economía Crítica, n. ${ }^{\circ}$ 20, Segundo semestre, ISNN 2013-5254.

Kenworthy, L, (2001). «Wage-Setting Measures. A survey and assessment». World Politics. 54.1, October 2001, 57-98

Kessler, I. and Purcell, J. (1992). «Performance related pay: objectives and application». Human Resource Management Journal. Vol.2 N.3.

Pendleton, A. Whitfield, K., Bryson, A.(2009). «The changing use of contingent pay at the modern British workplace». The Evolution of the Modern Workplace. Cambridge University Press.

Perry, J. L., Engbers, T. A., \& Jun, S. Y. (2009). Back to the Future? Performance-Related Pay, Empirical Research, and the perils of Resistance. Public Administration Review, 69 (1): 33-51.

Pouliakas, K., Theodoropoulos, N. (2011). «The Effect of Variable Pay Schemes on Workplace Absenteeism». IZA DP N. ${ }^{\circ} 5941$.

Ramos R., Sanromá E., Simón H. (2014). «Public-Private sector wage Differentials by type of contract: evidence from Spain». Document de Treball XREAP2014-08

Ruesga Benito, S.M. (2015): «Elementos de Reflexión Económica en el Análisis de Resultados de la Reforma Laboral de 2012». Temas para el debate. Fundación Sistema.

Schuler, R.S. (1998). Personnel and Human Resources Management. (3rd Edition). Toronto: West Publishing Company.

Suff P., and Reilly P. (2004). «Flexing your Remuneration: Variable Pay at Work». Institute for Employment Studies. UK.

VAN het KaAR, R. I Grünell M. (2001). «Variable pay in Europe». EIRO (European Industrial Relations Observatory).

Visser, J (2013). «Data Base on Institutional Characteristics of Trade Unions, Wage Setting, State Intervention and Social Pacts, 1960-2011 (ICTWSS)». Amsterdam Institute for Advanced Labour Studies AIAS University of Amsterdam. 